\title{
State-dependent fluorescence yields through the core-valence Coulomb exchange parameter
}

\author{
Piter S. Miedema, ${ }^{1, *}$ Philippe Wernet ${ }^{1}$ and Alexander Föhlisch ${ }^{1,2}$ \\ ${ }^{1}$ Institute for Methods and Instrumentation for Synchrotron Radiation Research G-ISRR, \\ Helmholtz-Zentrum für Materialien und Energie GmbH, \\ Albert-Einstein-Strasse 15, 12489 Berlin, Germany \\ ${ }^{2}$ Fakultät für Physik und Astronomie, Universität Potsdam, \\ Karl-Liebknecht-Strasse 24-25, 14476 Potsdam, Germany
}

(Dated: July 1, 2014)

\begin{abstract}
Total and partial fluorescence yield (PFY) L-edge x-ray absorption spectra differ from the transmission x-ray absorption spectra (XAS) through state-dependent fluorescence yield across the XAS. For $3 \mathrm{~d}^{1}$ to $3 \mathrm{~d}^{9}$ in octahedral symmetry we apply simulations of PFY and XAS and show how the atomic 2 p3d Coulomb exchange parameter $G_{p d}$ governs the differences in the $\mathrm{L}_{3} /\left(\mathrm{L}_{2}+\mathrm{L}_{3}\right)$ branching ratio between PFY and XAS. $\mathrm{G}_{\mathrm{pd}}$ orders the XAS final states following Hund's rules creating a strong state-dependent fluorescence decay strength variation across the XAS leading to the differences between PFY and XAS.
\end{abstract}

PACS numbers: 32.30.Rj, 31.15.vj, 78.70.En, 78.70.Dm

\section{INTRODUCTION}

To determine electronic structure and low energy excitations in matter, element-specific and chemically selective probes like x-ray absorption and inelastic x-ray scattering are uniquely powerful $[1,2]$. Radiative and non-radiative decay channels are of paramount importance for the partial fluorescence yield (PFY), total fluorescence yield (TFY) and electron-yield detection since they create the link between matter in any aggregate state and the true x-ray absorption coefficients of the Lambert-Beer law.

There is a distinction between hard $\mathrm{x}$-ray and soft $\mathrm{x}$ ray spectroscopy. Hard x-rays have a deeper probing depth and a sample under hard $\mathrm{x}$-ray radiation suffers less from beam damage due to the lower absorption cross section. However, the core levels in the soft x-ray regime are the sharpest available and therefore lead to a potentially higher resolution in the spectra and to the ultimate chemical resolution [3]. Thus, from the spectroscopy point of view, the soft x-ray regime is necessary for the most optimal electronic structure information. One important detail is that $3 \mathrm{~d}$-transition metal $\mathrm{L}_{2,3}$ (2p core)-edges suffer from $2 \mathrm{p} 3 \mathrm{~d}$ and $3 \mathrm{~d} 3 \mathrm{~d}$ multiplet effects, which at first would seem to complicate the understanding, but also allow a more detailed investigation of the valence and conduction band properties of these materials [4].

Another complicating factor in the soft x-ray regime is that, due to the small probing depth of soft x-rays, the true transmission $\mathrm{x}$-ray absorption is only accessible on highly tailored samples of nanometer thickness. That is why decay products (Auger electrons, fluorescence) of the x-ray absorption process are often taken as the measure for $\mathrm{x}$-ray absorption for samples that do not fulfill these thickness requirements. Note that in order to use the decay as a measure for the x-ray absorption, the decay has to be linearly proportional to the absorption cross section. Whereas electron-yield de- tection is surface-sensitive, TFY and PFY detection are well suited for condensed matter as highly bulk-sensitive probes. Extraction of electronic structure information from TFY and PFY spectral features requires accounting for x-ray optical effects [5], radiative and non-radiative transition matrix elements $[6,7]$ and dynamic screening involving charges beyond the atomic localization of the core-excited impurity final state [8, 9]. For TFY, the role of the background fluorescence is important as well as the possibility that a fluorescent photon is re-absorbed in the material. These are respectively called saturation and self-absorption effects of fluorescence yield probing.

The role of delocalization and electron transfer processes in TFY and PFY detected x-ray absorption has recently been under debate [10-15] proving that speculations [16] severely overestimated charge delocalization based on the assignment of spectral features derived from $\mathrm{x}$-ray optics $[11,17]$ to charge transfer processes instead. Work on a selected model system with comprehensive experimental x-ray spectroscopic measurements, ab initio restricted active space self consisted field (RAS-SCF) for core-excited states in direct comparison to semi-empirical crystal field multiplet (CFM) has identified the statedependent fluorescence yield from the atomic dipole transition matrix elements as the crucial parameter to describe TFY and PFY in a rather localized atomic picture [17]. This also shows that the assumption of fluorescence decay to be linearly proportional to the absorption cross section is not rock-solid true, but nevertheless may still be valid to some extent.

In this research paper we demonstrate for $3 \mathrm{~d}^{1}-3 \mathrm{~d}^{9}$ configurations of di- and/or tri-valent transition metal ions in octahedral $\left(\mathrm{O}_{\mathrm{h}}\right)$ symmetry how the $2 \mathrm{p} 3 \mathrm{~d}$ Coulomb exchange term $\mathrm{G}_{\mathrm{pd}}$ governs state-dependent fluorescence yields. Because $G_{p d}$ is largely responsible for the relative position of XAS final states (Note: XAS final states $=$ PFY intermediate states) within a spin-orbit manifold [18], coupling of this ordering of XAS final J- 
states with the electronic dipole transition rule $\Delta \mathrm{J}= \pm 1,0$ (for $\Delta \mathrm{J}=0, \mathrm{~J}_{\text {initial }} \neq 0$ ), leads to different fluorescence strengths over the XAS edge: ordering of the relative energy of J-states explains why the high spin states with high (low) J-multiplicity at the low-energy side of the $\mathrm{L}_{3}$ edge for systems with more (less) than half-filled 3d-shells have on average smaller decay strengths than XAS final states at higher energy in the $\mathrm{L}_{3^{-}}$and $\mathrm{L}_{2}$-edge. Thus a unified view on interpretation of TFY and PFY detected $\mathrm{XAS}$ techniques is given.

\section{THEORETICAL SECTION}

For $\mathrm{L}_{2,3}$-edge $\mathrm{x}$-ray absorption calculations on $3 \mathrm{~d}$ transition metal compounds, the standard (semiempirical) calculation method is based on an atomic multiplet theory computer code developed by Cowan and described by him in [19].

\section{A. Crystal Field Multiplet Theory}

The group of Theo Thole (TT) extended the Cowan code to crystal field allowing calculations in any symmetry by including Phil Butler's code [4] and we refer to this extension to the Cowan code as the TT-multiplet program. Note that also other extensions, for example to compensate for covalency (charge transfer multiplet), are present in this program. Recently, user-friendly interfaces for the TT-multiplet program have become available, the CTM4XAS and CTM4RIXS interfaces [20]. For most results of calculations shown in this research we have used these interfaces, unless stated otherwise (see for example the subsection atomic multiplet calculations). This crystal field multiplet (CFM) model has been successful for over 25 years for simulating spectra that correspond to experiment for both XAS and PFY $[4,21]$.

Normal, e.g. transmission, x-ray absorption spectra of $\mathrm{Ti}^{3+}, \mathrm{Ti}^{2+}, \mathrm{Cr}^{3+}, \mathrm{Mn}^{3+}, \mathrm{Fe}^{3+}, \mathrm{Mn}^{2+}, \mathrm{Fe}^{2+}, \mathrm{Co}^{2+}, \mathrm{Ni}^{2+}$ and $\mathrm{Cu}^{2+}$ ions in $\mathrm{O}_{\mathrm{h}}$ were simulated using the CTM4XAS interface. Common empirical $\mathrm{O}_{\mathrm{h}}$ crystal field values of $10 \mathrm{Dq}=1.8(10 \mathrm{Dq}=1.2) \mathrm{eV}$ for all trivalent (divalent) ions were used [21]. Corresponding partial-fluorescence yield (PFY) spectra using the same crystal filed value of $10 \mathrm{Dq}=1.8(1.2) \mathrm{eV}$ for all trivalent (divalent) ions were calculated according Ref. [22]. In short, the combination of $\mathrm{L}_{2,3}$-edge XAS and the following (resonant) emission or fluorescence was taken into account, or in x-ray spectroscopy terms, the $2 \mathrm{p} 3 \mathrm{~d}$ resonant inelastic x-ray scattering (RIXS) calculation was performed using the same octahedral field as for the normal XAS. Following this 2p3d RIXS calculation, the CTM4RIXS interface was used to construct the RIXS map and the total emission per excitation energy was summed up, which results in the $3 \mathrm{~d}$ PFY-XAS.
For one particular situation, $\mathrm{Fe}^{3+}\left(3 \mathrm{~d}^{5}\right)$, besides calculations with a crystal field of $10 \mathrm{Dq}=1.8 \mathrm{eV}\left(\mathrm{Fe}^{3+}\right.$ in highspin (HS) configuration), calculations with a crystal field of $10 \mathrm{Dq}=4.0 \mathrm{eV}\left(\mathrm{Fe}^{3+}\right.$ in low-spin (LS) configuration) were performed to study the spin influence on differences between XAS and PFY.

For all different ions mentioned above calculations were performed with the exchange Coulomb $\mathrm{G}_{\mathrm{pd}}$ and direct Coulomb Slater integrals $F_{p d}$ and $F_{d d}$ and as well calculations were performed where combinations of these Slater integrals were set to zero. The different calculations are coded as $\left[\mathrm{G}_{\mathrm{pd}} \mathrm{F}_{\mathrm{pd}} \mathrm{F}_{\mathrm{dd}}\right]$, where 1 corresponds to inclusion of the atomic Slater integral value and 0 to neglect of the Slater integral. The multiplet calculations provide x-ray energy versus intensity in so-called sticks. These sticks are visually the vertical lines representing the intensity corresponding to a particular x-ray transition at a particular x-ray energy. This means as well that the sticks contain the information of the initial and final state of the x-ray transition. For reproducing real $\mathrm{x}$-ray spectra these sticks are broadened with a combination of lorentzian and gaussian broadening to reproduce experimental broadening due to core hole lifetimes and instrumental broadening. In all cases presented here (including the atomic multiplet calculations discussed in the next subsection), both the gaussian and lorentzian broadening were set to $0.2 \mathrm{eV}$. The lorentzian broadening of 0.2 $\mathrm{eV}$ is to account for the core hole lifetimes of the L-edges of 3d-materials [23], while the gaussian broadening of 0.2 $\mathrm{eV}$ was set larger than the best instrumental resolution of monochromators nowadays.

\section{B. Atomic multiplet calculations}

The TT-multiplet RCG-program was used for atomic XAS calculations $\left(2 \mathrm{p}^{6} 3 \mathrm{~d}^{\mathrm{n}}\right.$ to $2 \mathrm{p}^{5} 3 \mathrm{~d}^{\mathrm{n}+1}$ without crystal field) to analyze the contribution of different XAS final J-states to the spectrum. Corresponding atomic PFY calculations were done with $10 \mathrm{Dq}=0 \mathrm{eV}$ still according Ref. [22] (the method also shortly described above). For these atomic multiplet calculations, the effect of $\mathrm{G}_{\mathrm{pd}}$ is tested: the calculations were performed with $\mathrm{G}_{\mathrm{pd}}$ [111] and without $G_{p d}[011]$ while the other Slater integrals $\left(\mathrm{F}_{\mathrm{pd}} \mathrm{F}_{\mathrm{dd}}\right)$ were taken into account.

Note that both the CTM4XAS and CTM4RIXS interfaces as well as the atomic code used for the XAS and PFY calculations, TT-multiplet, throughout this paper can be downloaded from the CTM4XAS website [24]. In addition, the used atomic Cowan modified code for XAS calculations, TT-multiplet, is currently still available from [25]. 


\section{RESULTS}

\section{A. Fe $\mathrm{L}_{2,3}$-edge $\mathrm{XAS}$ and PFY versus Slater integrals}

We start with showing an example for the XAS and PFY calculations with and without some of the Slater integrals. FIG.1 shows the XAS (red dashed) and PFY (black solid) calculations of the $\mathrm{L}_{2,3}$-edge of $\mathrm{Fe}^{3+}$ with (a) and without (b) the Slater integrals and with combinations of Slater integrals (FIG.1 (c) to (h)). The calculations shown in FIG.1(a) agree reasonably well with experimental data from [12], while differences in the ratio of the first two peaks in XAS and PFY between experiment and calculations depends on the choice of $10 \mathrm{Dq}$ (which we have fixed in the present study to $1.8 \mathrm{eV}$ for trivalent ions and $1.2 \mathrm{eV}$ for divalent ions). The panels on the left in FIG.1 are all calculations that include $\mathrm{G}_{\mathrm{pd}}([1 \mathrm{xx}]$, while the panels on the right are all XAS and PFY calculations without $\mathrm{G}_{\mathrm{pd}}([0 \mathrm{xx}])$. At a first glance one may notice that the strong differences between the PFY and XAS occur in the left panels, thus when $\mathrm{G}_{\mathrm{pd}}$ is taken into account. While all the XAS and PFY are normalized on the highest peak in the $\mathrm{L}_{2}$-edge, it is recognized that the PFY has much lower intensity in the $\mathrm{L}_{3}$-edge compared to XAS in case the $\mathrm{G}_{\mathrm{pd}}$ is taken into account in the calculations.

Concerning the shape of the spectrum, XAS and PFY do not differ. For other ions, similar figures with and without Slater integrals were obtained and also for those the shape of the XAS and PFY with the same coding $\left[G_{p d} F_{p d} F_{d d}\right]$ does not differ. For more information on the XAS and PFY spectra of these other ions, you may directly contact us. Kurian et al. also find that the $\mathrm{L}_{3} /\left(\mathrm{L}_{2}+\mathrm{L}_{3}\right)$ intensity branching ratio $(\mathrm{BR})$ is different for XAS and PFY, and at the same time that the shape of the spectra is similar [22]. While the shape of the XAS and PFY are similar, we focus on the intensity ratios between the $\mathrm{L}_{3}$ and $\mathrm{L}_{2}$ edge for XAS and PFY.

In the following we use the $\mathrm{L}_{3} /\left(\mathrm{L}_{2}+\mathrm{L}_{3}\right)$ intensity branching ratio (BR) as the measure for intensity ratios. Thereafter we will have a closer look on the PFY vs XAS intensity differences as a function of energy within the $\mathrm{L}_{3}$-edge.

\section{B. L $\mathrm{L}_{2,3}$-edge XAS and 3d-PFY and PFY/XAS ratio for the $3 \mathbf{d}^{\mathrm{n}}$}

FIG.2(a) and FIG.2(b) present the analysis of the BR for PFY and XAS respectively over the whole 3d-series for calculations with and without (some of) the Slater integrals. For calculations with $\mathrm{G}_{\mathrm{pd}}=1$ (closed symbols), the PFY BR in FIG.2(a) increases with increasing 3dcount, while with $G_{p d}=0$ (open symbols), the PFY BR is relatively constant between 0.6 and 0.85 over the whole $3 \mathrm{~d}$ series. Similar behavior with and without the atomic $\mathrm{G}_{\mathrm{pd}}$ value is obtained for XAS BR in FIG.2(b), but over-

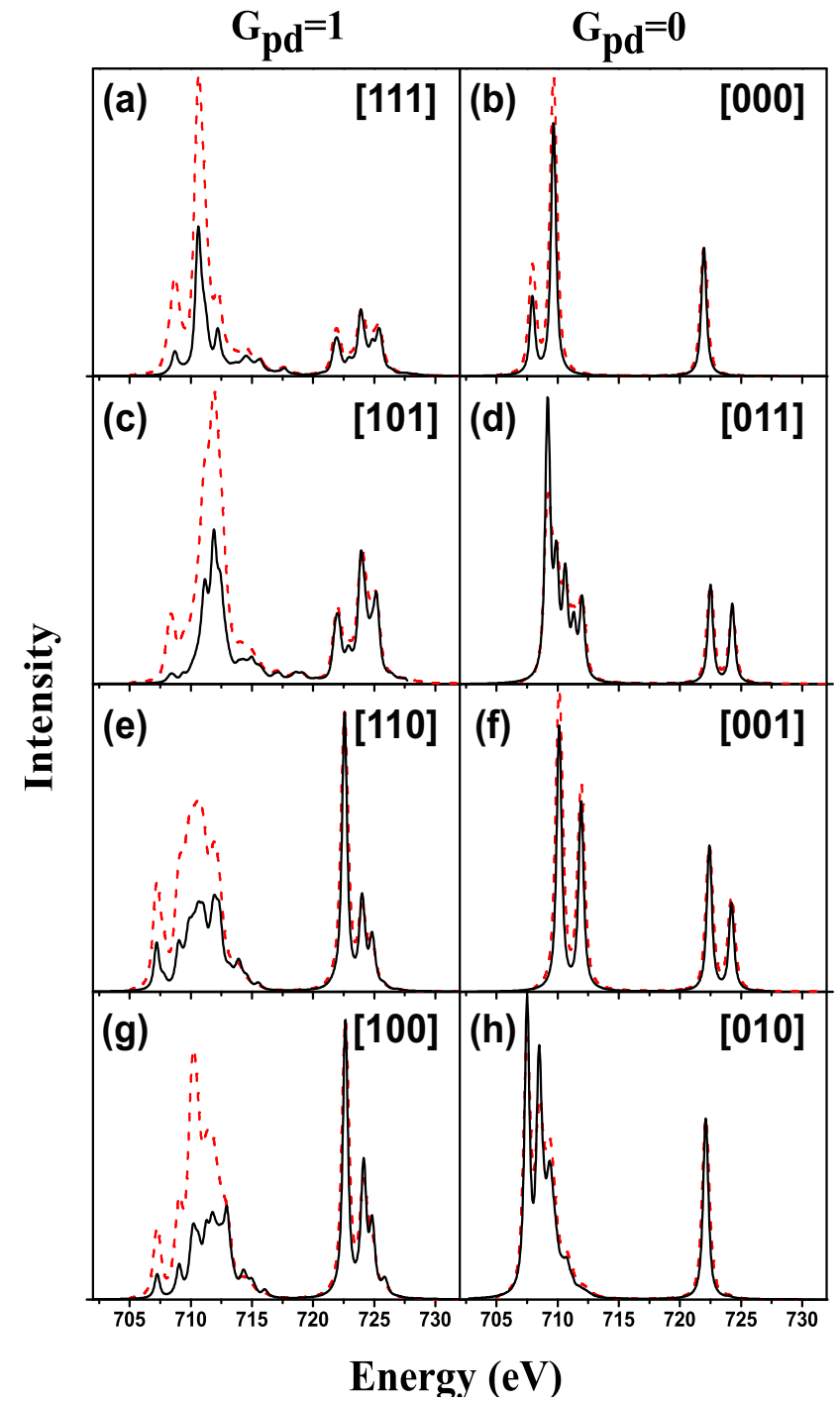

FIG. 1. (Color online) Calculated $\mathrm{Fe} \mathrm{L}_{2,3}$-edge XAS (red dashed) and PFY (black solid) for $\mathrm{Fe}^{3+}$ in $\mathrm{O}_{\mathrm{h}}$ with $10 \mathrm{Dq}=1.8$ eV (HS) including (a) all Slater integrals ([111]), (b) no Slater integrals [000], (c) without $\mathrm{F}_{\mathrm{pd}}$ [101], (d) without $\mathrm{G}_{\mathrm{pd}}$ [011], (e) without $\mathrm{F}_{\mathrm{dd}}$ [110], (f) without $\mathrm{F}_{\mathrm{pd}}$ and $\mathrm{G}_{\mathrm{pd}}$ [001], (g) without $F_{p d}$ and $F_{d d}[100]$ and $(h)$ without $G_{p d}$ and $G_{d d}[010]$ Slater integrals. Note that the panels (a), (c), (e) and (g) present the results of calculations with $\mathrm{G}_{\mathrm{pd}}=1$, while panels (b), (d), (f) and (h) show the XAS and PFY calculation results without $\mathrm{G}_{\mathrm{pd}}\left(\mathrm{G}_{\mathrm{pd}}=0\right)$. XAS and PFY are normalized on the highest $\mathrm{L}_{2}$ peak.

all the BR range is smaller for the XAS calculations. FIG.2(c) presents the PFY/XAS BR ratios. With $\mathrm{G}_{\mathrm{pd}}=1$ (closed symbols) this $\mathrm{PFY} / \mathrm{XAS}$ ratio deviates significantly from one (which means PFY and XAS differ strong in the $\mathrm{L}_{3}$-edge) in the beginning of the $3 \mathrm{~d}$ series and approaches one for the $3 \mathrm{~d}^{\mathrm{n}}$ with $n \geq 7$. The PFY/XAS ratios of calculations with $\mathrm{G}_{\mathrm{pd}}=0$ (open symbols) are around one for the whole $3 \mathrm{~d}$ series, thus there is almost no BR difference between PFY and XAS for $G_{p d}=0$. Summarized, FIG.2 displays that $\mathrm{G}_{\mathrm{pd}}$ is the major contribut- 


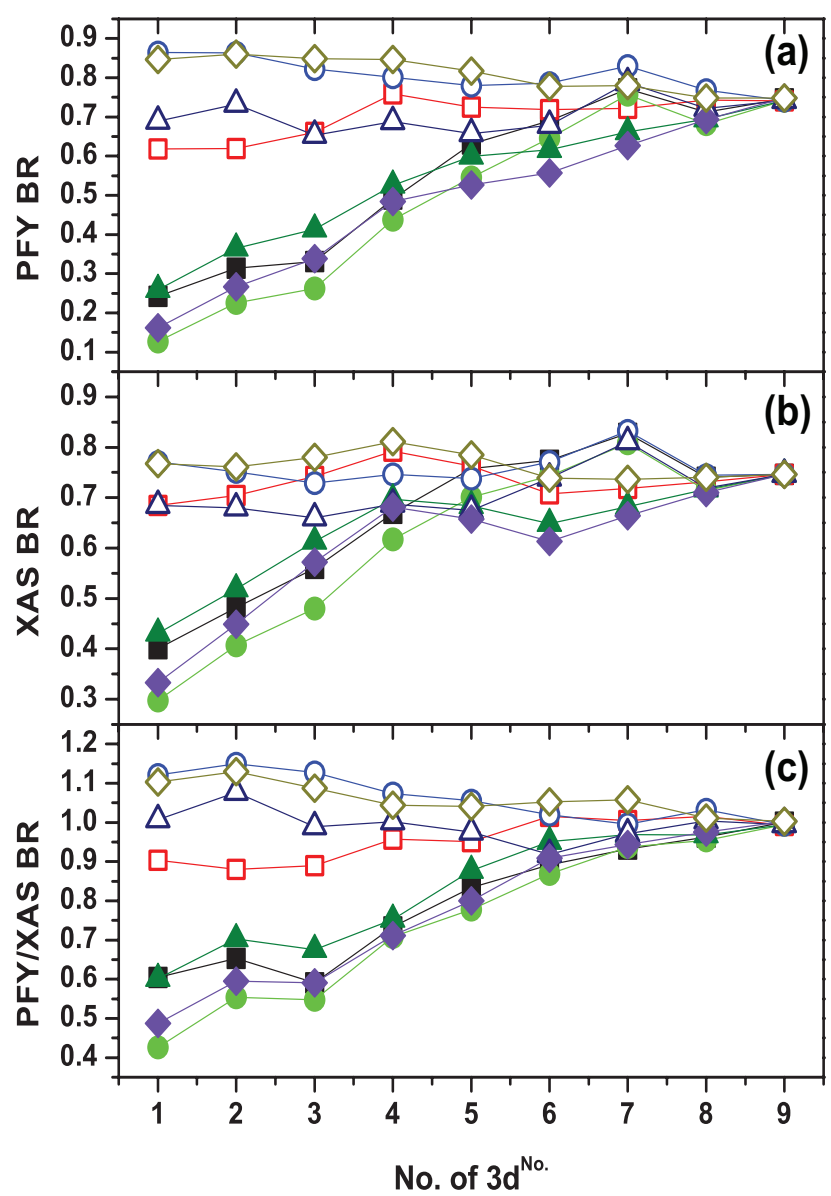

$G_{p d}=F_{p d}=F_{d d}=1[111]-F_{p d}=0[101]-F_{d d}=0[110]-F_{p d}=F_{d d}=0[100]$ $G_{p d}=F_{p d}=F_{d d}=0[000]-O-G_{p d}=0[011]-\triangle G_{p d}=F_{p d}=0[001]-\checkmark-G_{p d}=F_{d d}=0[010]$

FIG. 2. (Color online) $\mathrm{L}_{3} /\left(\mathrm{L}_{2}+\mathrm{L}_{3}\right)$ intensity branching ratio (BR) versus $3 \mathrm{~d}^{\mathrm{n}}$ for: (a) PFY and (b) XAS. (c) BR ratio between PFY/XAS. All panels show the BR results for calculations where all Slater integrals were included as well as BRs of calculations with exclusion of some (or all) of the Slater integrals. For all panels, the closed symbols indicate the calculations with $\mathrm{G}_{\mathrm{pd}}=1$.

ing factor for PFY and XAS BR differences.

\section{PFY and XAS as function of spin}

In the following we discuss the effects of the $G_{p d}, F_{p d}$ and $F_{d d}$ Slater interactions on PFY and XAS BRs for $\mathrm{Fe}^{3+}$ high-spin (HS) and low-spin (LS) and the effects of $\mathrm{G}_{\mathrm{pd}}$ on atomic XAS and PFY calculations. $\mathrm{Fe}^{3+}$ calculations with HS (red (gray)) and LS (black) configurations and with and without $\mathrm{G}_{\mathrm{pd}}, \mathrm{F}_{\mathrm{pd}}$ and $\mathrm{F}_{\mathrm{dd}}$ were analyzed resulting in the PFY (FIG.3(a)) and XAS (FIG.3(b)) BR analysis and the PFY/XAS BR ratios (FIG.3(c)), where

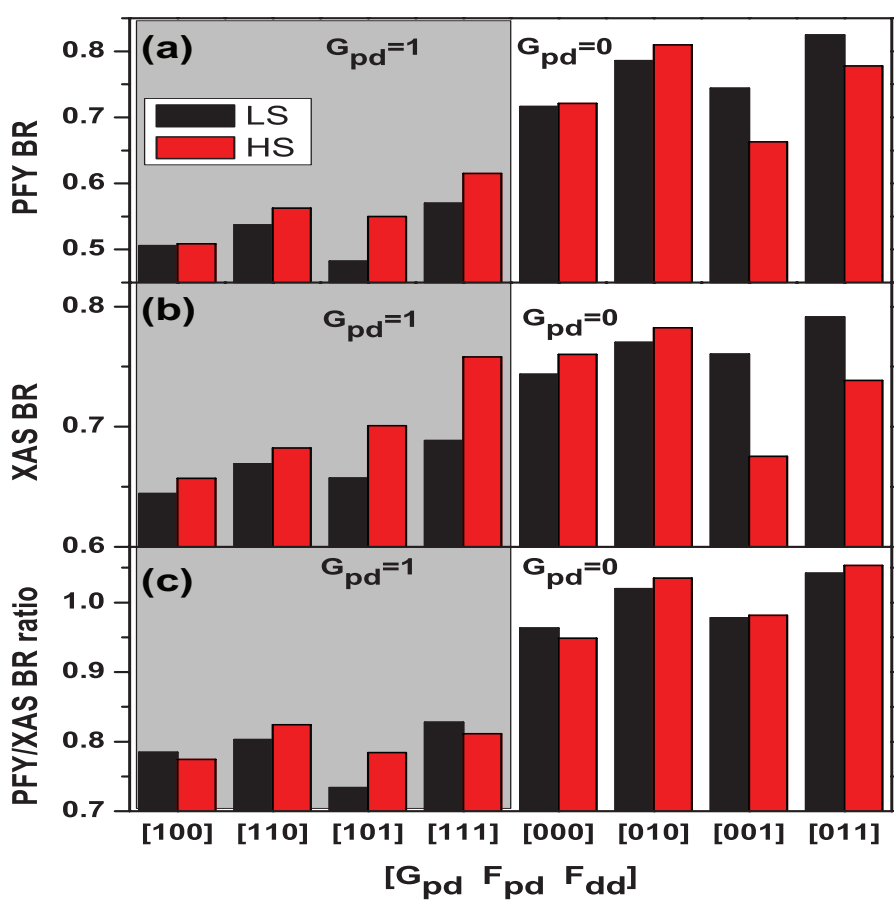

FIG. 3. (Color online) The BR of: (a) PFY, (b) XAS and (c) the $\mathrm{PFY} / \mathrm{XAS} \mathrm{BR}$ ratio versus the $\mathrm{Fe}^{3+}$ calculations with coding $\left[\begin{array}{lll}G_{p d} & F_{p d} & F_{d d}\end{array}\right]$ (1=atomic value, $0=$ neglect of respective Slater integral). Gray areas indicate calculations with $\mathrm{G}_{\mathrm{pd}}=1$. Note the BR range differences for panel (a) and (b). Red and black bars correspond to calculations for $\mathrm{Fe}^{3+}$ highspin (HS) and low-spin (LS) respectively.

the horizontal axes present the $\left[G_{p d} F_{p d} F_{d d}\right]$ calculations coding. The PFY BR (FIG.3(a)) for calculations with $G_{p d}=1$ is lower than with $G_{p d}=0$ and the PFY BRs of calculations with $\mathrm{F}_{\mathrm{pd}}=1$ are higher than the ones without. Hence $F_{p d}$ is slightly counteracting the $G_{p d}$ effect to the PFY BR. PFY BRs of $\mathrm{Fe}^{3+} \mathrm{LS}$ (black) for calculations with $F_{d d}=1$ decrease stronger when $G_{p d}=1$ as well, creating the large differences between the HS and LS BRs. For calculations with $\mathrm{G}_{\mathrm{pd}}=0$ and $\mathrm{F}_{\mathrm{dd}}=1$, the PFY BR of $\mathrm{Fe}^{3+}$ LS exceeds the PFY BR of $\mathrm{Fe}^{3+}$ HS. All these trends are also visible in the XAS BR, however there is a BR range difference between FIG.3(a) and FIG.3(b). It is known for $\mathrm{L}_{2,3}$-edge XAS that the $\mathrm{Fe}^{3+}$ HS has a larger BR than $\mathrm{Fe}^{3+}$ LS [26] but with $\mathrm{G}_{\mathrm{pd}}=0$ and at least $\mathrm{F}_{\mathrm{dd}}=1$ taken into account in the XAS calculation, the $\mathrm{Fe}^{3+}$ LS XAS BR is higher than the HS XAS BR (FIG.3B). The XAS BRs for $\mathrm{Fe}^{3+}$ HS and LS differ stronger when $F_{d d}=1$ : with both $G_{p d}=1$ and $F_{d d}=1$ the difference between the XAS BR of HS (red) and LS (black) is strong ([101] and [111]) with HS having a larger BR than LS. In cases with $\mathrm{G}_{\mathrm{pd}}=0$, while $\mathrm{F}_{\mathrm{dd}}=1$ ([001] and [011]) the XAS BR for LS exceeds the BR for HS as obtained before for the PFY calculations. Finally, the $\mathrm{F}_{\mathrm{pd}}=1$ cases counteract the BR lowering by $\mathrm{G}_{\mathrm{pd}}$ as was also seen before for the PFY calculations.

The PFY/XAS BR ratio in FIG.3(c) summarizes the observations on different spin configurations. With 
$\mathrm{G}_{\mathrm{pd}}=0$ the PFY $/ \mathrm{XAS}$ ratio is close to one. With $\mathrm{F}_{\mathrm{pd}}=1$ the PFY/XAS BR ratio increases for both $\mathrm{Fe}^{3+} \mathrm{HS}$ and LS compared to calculations with $\mathrm{F}_{\mathrm{pd}}=0$. Recapitulated, FIG.3 demonstrates that the $\mathrm{G}_{\mathrm{pd}}$ interaction plays the dominant role in $\mathrm{BR}$ differences between PFY and XAS for different spin (HS and LS) configurations as well, while the $F_{p d}$ interaction slightly counteracts the $G_{p d}$ based differences. The direct Coulomb term $\mathrm{F}_{\mathrm{dd}}$ acts stronger on the BR of $\mathrm{Fe}^{3+} \mathrm{LS}$, since the 3d-electrons in the LS configuration are closer packed than in the HS configuration, because only three of the five $3 \mathrm{~d}$-orbitals in LS are occupied, while for HS the 3d-electrons are separated among all the five orthogonal 3d-orbitals.

\section{DISCUSSION}

$\mathrm{Up}$ to here we have shown that $\mathrm{G}_{\mathrm{pd}}$ is responsible for the $\mathrm{L}_{3} /\left(\mathrm{L}_{2}+\mathrm{L}_{3}\right)$ BR differences between PFY and XAS. In addition, we have shown that this holds for different (HS and LS) spin configurations. We turn back to the left panels of FIG.1, the XAS and PFY calculations of $\mathrm{Fe}^{3+}$ with $\mathrm{G}_{\mathrm{pd}}=1$. For these panels there is a second trend: within the $\mathrm{L}_{3}$-edge there are differences between PFY and XAS comparing the low-energy side (with large intensity differences between PFY and XAS) and the high-energy side of the $\mathrm{L}_{3}$-edge (with relatively similar intensity for PFY and XAS). While we claimed above that $G_{p d}$ is responsible for the BR differences between $\mathrm{PFY}$ and XAS, the BR differences can be tracked back to the larger intensity differences at the beginning of the $\mathrm{L}_{3}$-edge. We want to stress that this observation is valid for all other ions studied. The question rises what effect the 2p3d Coulomb exchange $G_{p d}$ actually has to the XAS and PFY spectra. Van Veenendaal et al. claimed that $G_{p d}$ is largely responsible for the relative position of XAS final states within a spin-orbit manifold [18]. In order to understand this statement, we take back a step in the complexity of calculations and therefore we go from crystal field multiplet theory into atomic multiplet theory.

As an example PFY and XAS atomic spectra (calculated with atomic multiplet theory, see Theoretical Section, subsection B. Atomic multiplet calculations) are shown in FIG.4 for $\mathrm{Fe}^{3+}$ (left) and $\mathrm{Ni}^{2+}$ (right) together with the XAS final $2 \mathrm{p}^{5} 3 \mathrm{~d}^{\mathrm{n}+1} \mathrm{~J}$-state contributions for the case including all Slater Coulomb interactions (FIG.4(a) and FIG.4(b), [111]) and for $\mathrm{G}_{\mathrm{pd}}=0$ (FIG.4(c) and FIG.4(d), [011]). One cannot expect these atomic multiplet calculations to agree with experimental spectra (unless the experimental data are from single ions without surrounding as shown in [27]). Anyway, these atomic multiplet calculations will provide what the effect of $\mathrm{G}_{\mathrm{pd}}$ to XAS and PFY is. Comparing FIG.4(a) and FIG.4(b) with FIG.4(c) and FIG.4(d) respectively provides the effect of the $G_{p d}$ to the atomic spectra. FIG.4(a) and FIG.4(b) show that the intensity differences between PFY (magenta) and XAS (black dotted) appear

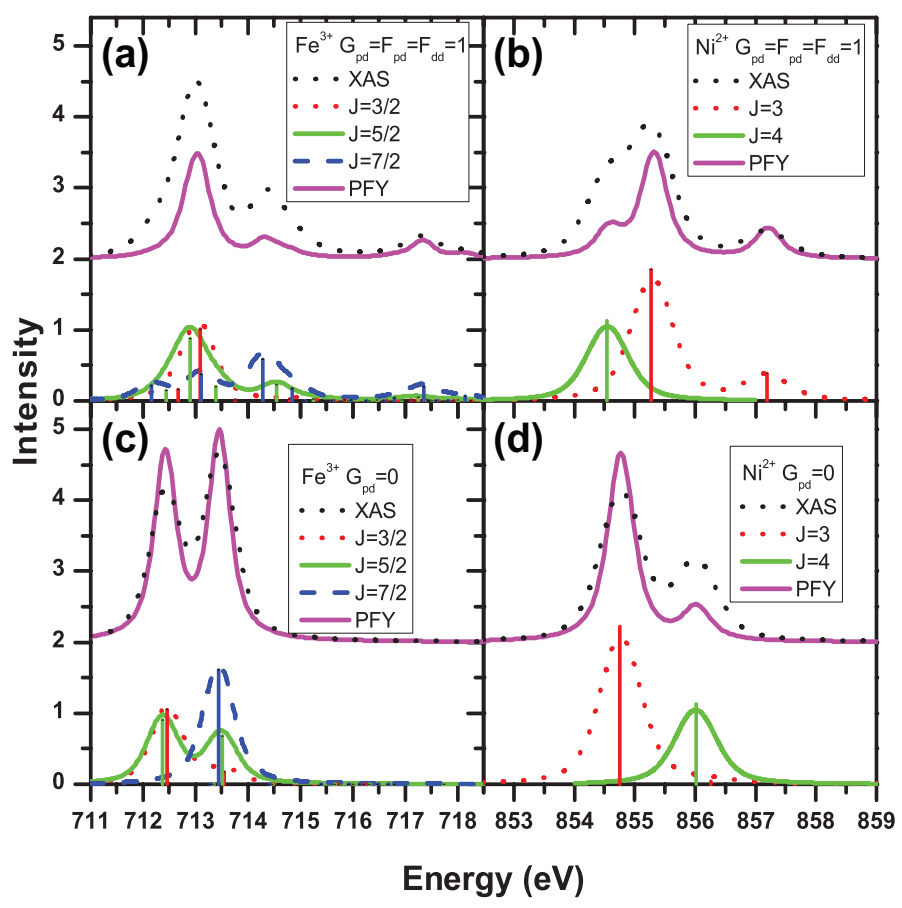

FIG. 4. (Color online) $\mathrm{L}_{3}$-edge atomic PFY (magenta solid) and XAS multiplet calculations (black dotted) on top of all panels of $\mathrm{Fe}^{3+}(\mathrm{a}, \mathrm{c})$ and $\mathrm{Ni}^{2+}(\mathrm{b}, \mathrm{d})$ together with, for (a) and (c) the $\mathrm{J}=3 / 2$ (red dotted), $\mathrm{J}=5 / 2$ (green solid) and $\mathrm{J}=7 / 2$ (blue dashed) final state contributions for $\mathrm{Fe}^{3+}$ and for (b) and (d) the $\mathrm{J}=3$ (red dotted) and $\mathrm{J}=4$ (green solid) XAS final state contributions for $\mathrm{Ni}^{2+}$. Panels (a) and (b) show calculations with all Slater integrals included ([111]), and panels (c) and $(\mathrm{d})$ show calculations with $\mathrm{G}_{\mathrm{pd}}=0([011])$. All spectra are normalized on the highest $\mathrm{L}_{2}$-peak not shown here. The full $\mathrm{L}_{2,3}$-edge spectra can be obtained from the authors directly.

mainly in the beginning and partially in the center of the $\mathrm{L}_{3}$-edge. Therefore $\mathrm{J}=7 / 2$ (blue dashed) and some $\mathrm{J}=5 / 2$ $(\mathrm{J}=4)$ (green solid) XAS final J-states that appear in the beginning and center of the $\mathrm{L}_{3}$-edge should have a lower fluorescent decay strength for $\mathrm{Fe}^{3+}\left(\mathrm{Ni}^{2+}\right)$ compared to other calculated sticks at the end of the $\mathrm{L}_{3}$-edge, where the intensities of PFY and XAS become similar.

With $\mathrm{G}_{\mathrm{pd}}=0$ only small intensity differences between XAS (black dotted) and PFY (magenta) remain (FIG.4(c) and FIG.4(d)). While the inclusion of $\mathrm{G}_{\mathrm{pd}}$ in the calculation drags the $\mathrm{J}=7 / 2(\mathrm{~J}=4)$ states (the highest J-states for respectively $\mathrm{Fe}^{3+}$ and $\mathrm{Ni}^{2+}$, red dotted) to lower energy for $\mathrm{Fe}^{3+}\left(\mathrm{Ni}^{2+}\right)$ as seen in FIG.4(a) (FIG.4(b)), for the calculations with exclusion of the $\mathrm{G}_{\mathrm{pd}}$ Slater integral, some of these highest J-states are not drawn to the low-energy side of $\mathrm{L}_{3}$-edge. In turn there is a slight shift of the other lower-J contributions to lower energy. In addition, the calculations without $\mathrm{G}_{\mathrm{pd}}$ lead to overlap of XAS transitions to different XAS final J-states at similar energies in the case of $\mathrm{Fe}^{3+}$, while in the case of $\mathrm{Ni}^{2+}$ the $\mathrm{J}=3$ states occur even only with one distinct stick. Because XAS (black, FIG.4(c) and FIG.4(d)) and PFY (pink, FIG.4(c) and FIG.4(d)) for $\mathrm{G}_{\mathrm{pd}}=0$ have al- 
most equal intensity and since there is a large overlap of the different XAS final J-states for $\mathrm{G}_{\mathrm{pd}}=0$ (or in the case of $\mathrm{Ni}^{2+}$ overlap of more $\mathrm{J}=3$ states at one energy), it is concluded that this overlap of different XAS transitions allows fluorescence decay through the fluorescent decay channel with higher decay strength which additionally leads to sharper PFY peak shapes. These were just two

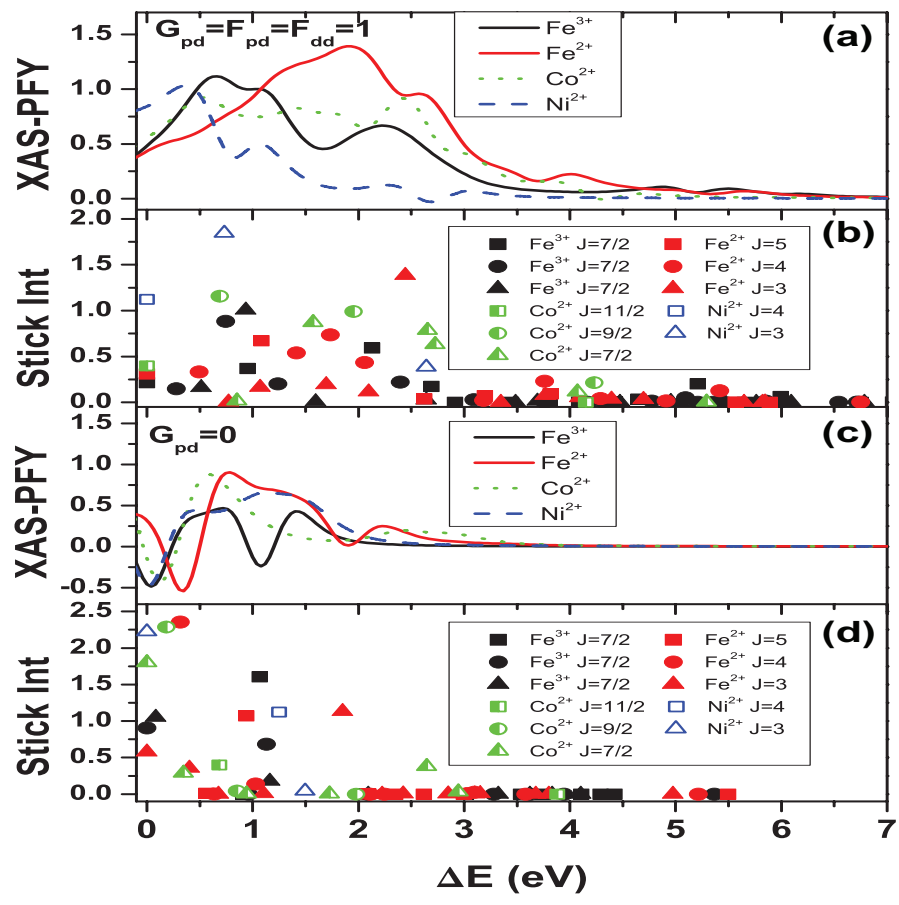

FIG. 5. (Color online) ((a) and (c)): Intensity difference between XAS and PFY at the $\mathrm{L}_{3}$-edge for atomic multiplet calculations of $\mathrm{Fe}^{3+}$ (black solid), $\mathrm{Fe}^{2+}$ (red (gray) solid), $\mathrm{Co}^{2+}$ (green dotted) and $\mathrm{Ni}^{2+}$ (blue dashed) with (a) all Slater integrals included and (c) with $\mathrm{G}_{\mathrm{pd}}=0$. All PFY and XAS spectra were normalized on the highest $\mathrm{L}_{2}$-edge peak. ((b) and (d)): Intensity of the calculated sticks with the final J-state of their $\mathrm{x}$-ray transition to the different XAS spectra with squares for the highest $J$, circles for the intermediate $\mathrm{J}$ and triangles for the lowest J-state contributions shown for calculations with (b) all Slater integrals included and (d) with $\mathrm{G}_{\mathrm{pd}}=0$. In panels (b) and (d) the black closed symbols refer to $\mathrm{Fe}^{3+}$, the red (gray) closed symbols to $\mathrm{Fe}^{2+}$, the half-filled green symbols to $\mathrm{Co}^{2+}$ and the open blue symbols refer to $\mathrm{Ni}^{2+}$. The horizontal axes for all panels present $\Delta \mathrm{E}$ defined as the energy difference between the spectrum or final J-state stick with the final J-state stick found at lowest energy.

examples of atomic XAS and PFY and in the following these observations are made more general by presenting the analysis for the atomic XAS and PFY calculations of $\mathrm{Fe}^{3+}$ to $\mathrm{Ni}^{2+}\left(3 \mathrm{~d}^{5}-3 \mathrm{~d}^{8}\right)$ ions. FIG.5(a) and FIG.5(c) show the difference between the XAS and PFY intensity (XAS-PFY) at the $\mathrm{L}_{3}$-edge for atomic multiplet calculations of the $\mathrm{Fe}^{3+}-\mathrm{Ni}^{2+}\left(3 \mathrm{~d}^{5}-3 \mathrm{~d}^{8}\right)$ ions with (a) all Slater integrals included and (c) with $\mathrm{G}_{\mathrm{pd}}=0$. The horizontal axis presents $\Delta \mathrm{E}$, which is defined as the energy difference with the energy of the stick at lowest energy. Thus at $\Delta \mathrm{E}=0$ appears the stick with lowest energy (as can be seen in FIG.5(b) and FIG.5(d)). We use this horizontal axis to add all the XAS-PFY in one figure, because the energies of the $\mathrm{L}_{3}$-edge XAS and PFY depend on the ion. FIG.5(a) shows that strong XAS-PFY differences occur at the beginning and middle of the $\mathrm{L}_{3}$-edge and these differences decrease over an energy range of $5 \mathrm{eV}$ to zero. There are also XAS-PFY differences with $\mathrm{G}_{\mathrm{pd}}=0$ (FIG.5(c)), but the absolute difference is lower than in FIG.5(a) and there are in addition negative differences. These positive and negative wiggles around a XAS-PFY difference show that with $G_{p d}=0$ the XAS and PFY are almost equal in intensity, while remaining differences are due to peak sharpness differences between PFY and XAS (FIG.4). Also over a much smaller energy range of $3 \mathrm{eV}$, the XAS-PFY differences are reduced entirely to zero for the calculations with $\mathrm{G}_{\mathrm{pd}}=0$.

FIG.5(b) and FIG.5(d) are used to relate the XAS$\mathrm{PFY}$ intensity differences to the $\mathrm{x}$-ray transitions and in particular to the final state of the x-ray transition. In other words the obtained XAS-PFY intensity differences can be traced to the different XAS final J-states represented by the calculated sticks. FIG.5(b) and FIG.5(d) show the calculated sticks with their stick intensity on the vertical axis and the final J-state that the stick belongs to. Squares represent the highest XAS final J-state values, circles represent intermediate final J-state values and triangles present the lowest final J-state values. The absolute value of the highest, intermediate and lowest Jstate values depends on the amount of electrons in the 3d-shell and is therefore different for all the ions. In FIG.5(b) the stick at $\Delta \mathrm{E}=0$ has for all four ions a XAS final J-state with highest J (squares). Then it is noticed that the highest XAS-PFY differences in FIG.5(a) are strongly connected to both XAS final J-states with highest $\mathrm{J}$ (squares) and intermediate $\mathrm{J}$ (circles) of the corresponding ion in FIG.5(b), while dips in the XASPFY intensity (thus dips in the difference between XAS and PFY spectra) in FIG.5(a) are related to the final Jstates with lowest $\mathrm{J}$ (triangles in FIG.5(b)) and in a few cases $\left(\mathrm{Fe}^{2+}\right.$, red (gray) closed symbols and $\mathrm{Co}^{2+}$, green half-filled symbols) in addition to intermediate J. Comparing the calculated sticks of FIG.5(b) with FIG.5(d) shows that the final J-state corresponding to the stick at $\Delta \mathrm{E}=0$ is not from the highest J-type anymore in case $\mathrm{G}_{\mathrm{pd}}=0$ (FIG.5(d)). Furthermore the XAS-PFY differences in FIG.5(c) cannot be related to a particular type of J-state and as said the XAS-PFY intensity differences in FIG.5(c) were related to difference in peak sharpness. Besides, the different sticks representing x-ray transitions to different J-states in FIG.5(d) are closer in energy and in intensity.

From FIGS. 4 and 5 and their analyses we conclude that $\mathrm{G}_{\mathrm{pd}}$ orders the XAS final J-states such that high J-states appear at the low-energy side of the $\mathrm{L}_{3}$-edge following Hund's rules applied to the $2 \mathrm{p}^{5} 3 \mathrm{~d}^{\mathrm{n}+1}$ configuration, confirming Ref. [18]. Hund's rules for the $2 \mathrm{p}^{6} 3 \mathrm{~d}^{\mathrm{n}}$ initial (PFY final) state provide that the state with the highest 
$\mathrm{S}$ and then the highest $\mathrm{L}$ and highest $\mathrm{J}$ (for the more than half-filled 3d-shell; for less than half-filled it is lowest J, for clarity we focus on the more than half-filled 3d-shell) becomes the ground state. Along with $2 \mathrm{p}^{5} 3 \mathrm{~d}^{\mathrm{n}+1}$ J-state ordering by $\mathrm{G}_{\mathrm{pd}}$, the transitions from the initial ground state with highest $\mathrm{S}$ and $\mathrm{J}$ to the final XAS J-states with lowest energies grouping up the high J-states at the lowenergy side of the $\mathrm{L}_{3}$-edge, also will have a relatively high $\mathrm{S}$ and $\mathrm{J}$ (again, this is when $\mathrm{G}_{\mathrm{pd}}=1$ ). For these final XAS states it will be more difficult to decay with fluorescence: since many of the J-states of the initial state (and the PFY final state) $3 \mathrm{~d}^{\mathrm{n}}$ term symbol states are out of reach for the final XAS states with high J due to the electronic dipole transition rules $\Delta \mathrm{J}= \pm 1,0$, e.g. many of the PFY final states (above the PFY final ground state) have lower J than can be reached following the dipole transition rules. Nevertheless, Auger decay from the XAS final states with highest $\mathrm{J}$ is not disturbed, thus transmission and electron-yield XAS will have a higher intensity at the lower side of the $\mathrm{L}_{3}$-edge than the corresponding fluorescence yield XAS. A similar reason holds for the ions with a less than half-filled 3d-shell: the lowest J-state is the ground state and with $G_{p d}=1$ the J-states with lowest $J$ are also the ones at lowest energy for $2 \mathrm{p}^{5} 3 \mathrm{~d}^{\mathrm{n}+1}$ and that prevents again an easy fluorescent decay from the low-energy side of the $\mathrm{L}_{3}$-edge.

In the present research, only $3 \mathrm{~d}-\mathrm{PFY}$ (in addition to $\mathrm{XAS}$ ) calculations were done, which is not the same as TFY, but it is an essential part of TFY and can be considered the major contributing part neglecting the background fluorescent decay (the latter could even lead to dips in the TFY spectrum and we believe that the low fluorescent decay at the low-energy side of the $\mathrm{L}_{3}$-edge may contribute to the observation of these dips). Since the $3 \mathrm{~d}-2 \mathrm{p}$ interaction is at least hundred times stronger than the $3 \mathrm{~s}-2 \mathrm{p}$ interaction, we may neglect metal $3 \mathrm{~s}$ PFY decay. The remaining difference between TFY and PFY is the aforementioned background fluorescence of the other present elements, which was analyzed in more detail before $[10,11,17]$.

\section{SUMMARY AND CONCLUSIONS}

In summary, the exchange Coulomb parameter $G_{p d}$ leads to different BR for XAS and PFY in the 3d series for $3 \mathrm{~d}^{\mathrm{n}}, \mathrm{n}<9 . \mathrm{G}_{\mathrm{pd}}$ is responsible for the relative energy position of the $2 \mathrm{p}^{5} 3 \mathrm{~d}^{\mathrm{n}+1}$ states and therefore for the distribution of these states over the $\mathrm{L}_{2,3}$-edge and especially the $\mathrm{L}_{3}$-edge XAS. In essence, $\mathrm{G}_{\mathrm{pd}}$ leads to XAS final Jstates at the low-energy side of the $\mathrm{L}_{3}$-edge which tend to have lower fluorescence strengths, while there is no influence on the major (Auger) decay channel from these XAS final states. In turn this explains BR differences between PFY on one side and transmission and electronyield detected XAS on the other side. In addition we have shown that $G_{p d}$ is for both HS and LS configurations the most important contribution to BR differences between PFY and XAS. In contradiction to Bokarev et al. [12] we show that the differences in PFY compared to XAS do not come from delocalization in combination with different radiative lifetimes. Since the core hole lifetimes are determined by the Auger decay in the soft $\mathrm{x}-$ ray regime [4] we oppose that statement and show that the differences depend entirely on the Coulomb exchange $\mathrm{G}_{\mathrm{pd}}$ parameter, which is in essence an ionic and local property.
* p.s.miedema@gmail.com

[1] J. Stöhr, NEXAFS Spectroscopy (Springer-Verlag, Berlin, 2003).

[2] L. J. P. Ament, M. van Veenendaal, T. P. Devereaux, J. P. Hill, and J. van den Brink, Rev. Mod. Phys. 83, 705 (2011).

[3] F. J. Himpsel, physica status solidi (b) 248, 292 (2011).

[4] F. M. F. de Groot and A. Kotani, Core Level Spectroscopy of Solids (Advances in Condensed Matter Science) (CRC Press, Boca Raton, FL, 2008).

[5] S. Eisebitt, T. Böske, J.-E. Rubensson, and W. Eberhardt, Phys. Rev. B 47, 14103 (1993).

[6] F. de Groot, M. Arrio, P. Sainctavit, C. Cartier, and C. Chen, Solid State Communications 92, 991 (1994).

[7] E. Suljoti, F. M. F. de Groot, M. Nagasono, P. Glatzel, F. Hennies, M. Deppe, A. Pietzsch, B. Sonntag, A. Föhlisch, and W. Wurth, Phys. Rev. Lett. 103, 137401 (2009).

[8] P. Nozières and C. T. De Dominicis, Phys. Rev. 178, 1097 (1969).

[9] G. D. Mahan, Phys. Rev. 163, 612 (1967).
[10] T. Z. Regier, A. J. Achkar, D. Peak, J. S. Tse, and D. G. Hawthorn, Nature Chemistry 4, 765 (2012).

[11] F. M. F. de Groot, Nature Chemistry 4, 766 (2012).

[12] S. I. Bokarev, M. Dantz, E. Suljoti, O. Kühn, and E. F. Aziz, Phys. Rev. Lett. 111, 083002 (2013).

[13] R. Green, D. Peak, A. Achkar, J. Tse, A. Moewes, D. Hawthorn, and T. Regier, Phys. Rev. Lett. 112, 129301 (2014).

[14] A. Föhlisch, F. de Groot, M. Odelius, S. Techert, and P. Wernet, Phys. Rev. Lett. 112, 129302 (2014).

[15] S. I. Bokarev, M. Dantz, E. Suljoti, K. Atak, B. Winter, O. Kühn, and E. F. Aziz, Phys. Rev. Lett. 112, 129303 (2014).

[16] E. F. Aziz, M. H. Rittmann-Frank, K. M. Lange, S. Bonhommeau, and M. Chergui, Nature Chemistry 2, 853 (2010).

[17] P. Wernet, K. Kunnus, S. Schreck, W. Quevedo, R. Kurian, S. Techert, F. M. F. de Groot, M. Odelius, and A. Föhlisch, The Journal of Physical Chemistry Letters 3, 3448 (2012).

[18] M. van Veenendaal, J. B. Goedkoop, and B. T. Thole, Phys. Rev. Lett. 77, 1508 (1996). 
[19] R. Cowan, The theory of atomic structure and spectra (University of California Press, Berkeley, 1981).

[20] E. Stavitski and F. M. de Groot, Micron 41, 687 (2010).

[21] P. S. Miedema, x-ray spectroscopy of inorganic materials, Ph.D. thesis, Utrecht University (2012).

[22] R. Kurian, K. Kunnus, P. Wernet, S. M. Butorin, P. Glatzel, and F. M. F. de Groot, Journal of Physics: Condensed Matter 24, 452201 (2012).

[23] G. Zschornack, Handbook of X-Ray Data (SpringerVerlag, Berlin Heidelberg, 2007).

[24] "Ctm4xas, http://www.anorg.chem.uu.nl/ctm4xas/, Access to downloads can be requested from f.m.f.degroot@uu.nl,”.

[25] "http:www.anorg.chem.uu.nl/people/staff/ FrankdeGroot/SLS/sls2007.htm, Accessed: 201404-16,".

[26] B. T. Thole and G. van der Laan, Phys. Rev. B 38, 3158 (1988).

[27] K. Hirsch, V. Zamudio-Bayer, J. Rittmann, A. Langenberg, M. Vogel, T. Möller, B. v. Issendorff, and J. T. Lau, Phys. Rev. B 86, 165402 (2012). 